\title{
Correction to: Rare variant analysis of 4241 pulmonary arterial hypertension cases from an international consortium implicates FBLN2, PDGFD, and rare de novo variants in $\mathrm{PAH}$
}

Na Zhu ${ }^{1,2+}$, Emilia M. Swietlik ${ }^{3 \dagger}$, Carrie L. Welch ${ }^{1+}$, Michael W. Pauciulo ${ }^{4,5+}$, Jacob J. Hagen ${ }^{1,2}$, Xueya Zhou $^{1,2}$, Yicheng Guo ${ }^{2}$, Johannes Karten ${ }^{6}$, Divya Pandya ${ }^{3}$, Tobias Tilly ${ }^{3}$, Katie A. Lutz ${ }^{4}$, Jennifer M. Martin, Carmen M. Treacy ${ }^{3}$, Erika B. Rosenzweig ${ }^{1}$, Usha Krishnan ${ }^{1}$, Anna W. Coleman ${ }^{4}$ Claudia Gonzaga-Jaureguii, Allan Lawrie ${ }^{9}$, Richard C. Trembath ${ }^{10}$, Martin R. Wilkins ${ }^{11}$, Regeneron Genetics Center ${ }^{8}$, PAH Biobank Enrolling Centers' Investigators ${ }^{12}$, NIHR BioResource for Translational Research - Rare Diseases ${ }^{13}$, National Cohort Study of Idiopathic and Heritable PAH ${ }^{14}$, Nicholas W. Morrell ${ }^{3,7,15,16 \dagger}$, Yufeng Shen ${ }^{2,17 \dagger}$, Stefan Gräf ${ }^{3,18,7 \dagger}$,

William C. Nichols ${ }^{4,5 \dagger}$ and Wendy K. Chung ${ }^{1,19,20^{*}+}$

\section{Correction to: Genome Med 13, 80 (2021)}

https://doi.org/10.1186/s13073-021-00891-1

It was highlighted that the original article [1] contained an error in the name of Claudia Gonzaga-Jauregui. It was incorrectly captured as Gonzaga-Juaregui. The original article has been updated.

\section{Author details}

'Department of Pediatrics, Columbia University Irving Medical Center, 1150 St. Nicholas Avenue, Room 620, New York, NY 10032, USA. ${ }^{2}$ Department of Systems Biology, Columbia University, New York, NY, USA. ${ }^{3}$ Department of Medicine, University of Cambridge, Cambridge Biomedical Campus, Cambridge, UK. ${ }^{4}$ Division of Human Genetics, Cincinnati Children's Hospital Medical Center, Cincinnati, OH, USA. ${ }^{5}$ Department of Pediatrics, University of

The original article can be found online at https://doi.org/10.1186/s13073021-00891-1.

* Correspondence: wkc15@columbia.edu

${ }^{+}$Na Zhu, Emilia M. Swietlik, Carrie L. Welch, Michael W. Pauciulo contributed equally to this work.

${ }^{\dagger}$ Nicholas W. Morrell, Yufeng Shen, Stefan Gräf, William C. Nichols and Wendy K. Chung jointly supervised the work.

'Department of Pediatrics, Columbia University Irving Medical Center, 1150

St. Nicholas Avenue, Room 620, New York, NY 10032, USA

${ }^{19}$ Herbert Irving Comprehensive Cancer Center, Columbia University Irving Medical Center, New York, NY, USA

Full list of author information is available at the end of the article
Cincinnati College of Medicine, Cincinnati, OH, USA. ${ }^{6} 42$ Genetics, Belfast, Ireland. ${ }^{7} \mathrm{NIHR}$ BioResource for Translational Research, Cambridge Biomedical Campus, Cambridge, UK. ${ }^{8}$ Regeneron Pharmaceuticals, New York, NY, USA. ${ }^{9}$ Department of Infection, Immunity and Cardiovascular Disease, University of Sheffield, Sheffield, UK. ${ }^{10}$ Department of Medical and Molecular Genetics, King's College London, London, UK. ${ }^{11}$ National Heart \& Lung Institute, Imperial College London, London, UK. ${ }^{12}$ www.pahbiobank.org, Cincinnati, $\mathrm{OH}$, USA. ${ }^{13}$ University of Cambridge and Cambridge University Hospitals NHS Foundation Trust, Cambridge Biomedical Campus, Cambridge, UK.

${ }^{14}$ www.ipahcohort.com, Cambridge, UK. ${ }^{15}$ Addenbrooke's Hospital NHS Foundation Trust, Cambridge Biomedical Campus, Cambridge, UK. ${ }^{16}$ Royal Papworth Hospital NHS Foundation Trust, Cambridge Biomedical Campus, Cambridge, UK. ${ }^{17}$ Department of Biomedical Informatics, Columbia University, New York, NY, USA. ${ }^{18}$ Department of Haematology, University of Cambridge, Cambridge Biomedical Campus, Cambridge, UK. ${ }^{19}$ Herbert Irving

Comprehensive Cancer Center, Columbia University Irving Medical Center, New York, NY, USA. ${ }^{20}$ Department of Medicine, Columbia University Irving Medical Center, New York, NY, USA.

Published online: 22 June 2021

Reference

1. Zhu N, et al. Rare variant analysis of 4241 pulmonary arterial hypertension cases from an international consortium implicates FBLN2, PDGFD, and rare de novo variants in PAH. Genome Med. 2021;13:80. https://doi.org/10.1186/ s13073-021-00891-1.

(c) The Author(s). 2021 Open Access This article is licensed under a Creative Commons Attribution 4.0 International License, which permits use, sharing, adaptation, distribution and reproduction in any medium or format, as long as you give appropriate credit to the original author(s) and the source, provide a link to the Creative Commons licence, and indicate if changes were made. The images or other third party material in this article are included in the article's Creative Commons licence, unless indicated otherwise in a credit line to the material. If material is not included in the article's Creative Commons licence and your intended use is not permitted by statutory regulation or exceeds the permitted use, you will need to obtain permission directly from the copyright holder. To view a copy of this licence, visit http://creativecommons.org/licenses/by/4.0/ The Creative Commons Public Domain Dedication waiver (http://creativecommons.org/publicdomain/zero/1.0/) applies to the data made available in this article, unless otherwise stated in a credit line to the data. 